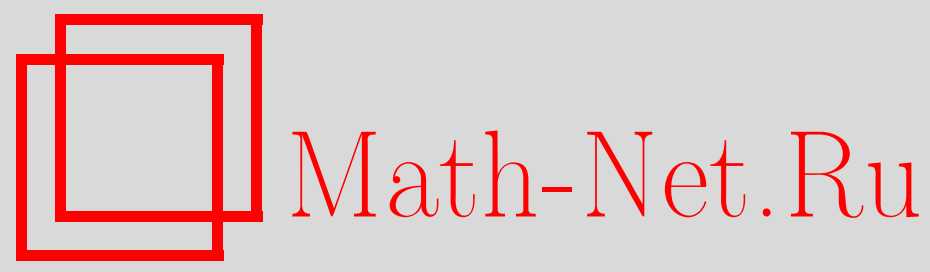

Е. Л. Александров, Спектральные функции самосопряженных и симметрических операторов умножения в пространствах $L^{2}(X, \mu)$, Матем. заметки, 2000, том 67, выпуск $6,803-810$

DOI: https://doi.org/10.4213/mzm898

Использование Общероссийского математического портала Math-Net.Ru подразумевает, что вы прочитали и согласны с пользовательским соглашением http://www . mathnet.ru/rus/agreement

Параметры загрузки:

IP : 54.224 .135 .184

26 апреля 2023 г., $14: 27: 19$

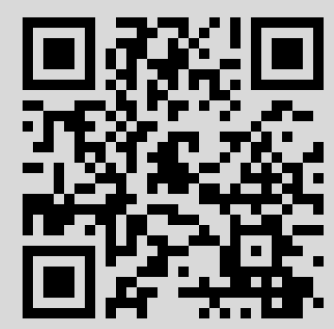




\section{СПЕКТРАЛЬНЫЕ ФУНКЦИИ САМОСОПРЯЖЕННЫХ И СИММЕТРИЧЕСКИХ ОПЕРАТОРОВ УМНОЖЕНИЯ В ПРОСТРАНСТВАХ $L^{2}(X, \mu)$}

\section{Е. Л. Александров}

Получены в явном виде формулы спектральной функции парного оператора умножения, содержащего мультипликативную инволюцию в пространстве $L^{2}(X, \mu)$, а также оператора типа свертки в пространствах $L^{2}\left(\mathbb{R}^{n}\right)$. Рассматриваются симметричные операторы умножения и свертки в пространствах $L^{2}(X, \mu)$ и $L^{2}\left(\mathbb{R}^{n}\right)$.

Библиография: 6 названий.

Хотя построение спектральной теории самосопряженных и симметрических операторов в гильбертовом пространстве можно считать завершенной, существует не так уж много операторов, для которых можно указать в явном виде их спектральные функции. Нахождение спектральных функций для конкретных операторов является важной и часто трудной задачей. Например, в квантовой механике наблюдаемая есть некоторьй самосопряженньй оператор $A$ в гильбертовом пространстве $\Phi$ состояний квантомеханической системы и, в отличие от классической механики, она (наблюдаемая) в состоянии $\varphi \in \Phi$ принимает не фиксированное значение, а является случайной величиной с распределением вероятностей $F(t)=\left(E_{t} \varphi, \varphi\right)$, где $E_{t}$ - спектральная функция оператора $A$, Знание $E_{t}$ в данном случае представляется важным.

В предлагаемой статье устанавливается спектральное разложение одного класса парных операторов умножения, содержащих инволюцию, в гильбертовом пространстве $L^{2}(X, \mu)$. Оператор $T$ этого класса имеет вид

$$
T f=\theta f+\eta U f, \quad f \in D(T),
$$

здесь $\theta, \eta$ - измеримые функции, заданные на пространстве $(X, \mu)$ с $\sigma$-конечной мерой $\mu$, $U$ - инволютивный оператор.

В случае, когда $T$ определен на максимально возможном линеале $D(T) \subset L^{2}(X, \mu)$ и самосопряжен, получена в явном виде его спектральная функция, т. е. семейство ортпроекторов $E_{t}, t \in \mathbb{R}=(-\infty, \infty)$, проектирующие на приводящие $T$ подпространства.

Рассматривается также случай, когда $T$ определен не на максимальном линеале и является симметрическим оператором с дефектными числами $(m, m)$.

Особо отметим случай, когда $X=\mathbb{R}^{n}$ и $\mu$-мера Лебега в $\mathbb{R}^{n}$. Операторы $T$ в этом случае унитарно эквивалентны операторам типа свертки в $L^{2}\left(\mathbb{R}^{n}\right)$, представляющим весьма широкий класс дифференциальных, интегро-дифференциальных, интегральных 
с ядрами, зависящими от разности и суммы аргументов, и других операторов, встречающихся во многих областях физики и математики. Укажем, например, проблему рассеивания энергии в атмосферах звезд и планет [1], кинетические уравнения которых являются интегральными с ядрами, зависящими от разности и суммы аргументов.

\section{1. Спектральные функции парных операторов умножения, содержащих мультипликативную инволюцию}

\section{1. Операторы умножения и мультипликативные инволюции.}

1. Пусть $H$ - гильбертово сепарабельное пространство со скалярным произведением $(\cdot, \cdot), A$ - самосопряженный оператор в $H$, определенньй на $D(A) \subset H$.

Напомним, что семейство ортпроекторов $E_{t}, t \in \mathbb{R}$, называется спектральной функиией оператора $A$, если оно удовлетворяет свойствам:

a) $E_{s} E_{t}=E_{\min \{s, t\}}, s, t \in \mathbb{R}$;

b) $E_{t-0}=E_{t}$;

c) $E_{-\infty}=O, E_{+\infty}=I$, где $O$ и $I-$ соответственно нулевой и тождественньй операторы в $H$;

d) $D(A)=\left\{f \in H: \int_{-\infty}^{\infty} t^{2} d\left(E_{t} f, f\right)<\infty\right\}$ и для каждого $f \in D(A)$

$$
A f=\int_{-\infty}^{\infty} t d E_{t} f
$$

Для любого интервала $\Delta=[\alpha, \beta]$ положим $E(\Delta)=E_{\beta+0}-E_{\alpha}$.

Теорема A [2]. Пусть $E_{t}, t \in \mathbb{R},-$ семейство ортпроекторов в $H$, удовлетворяющее a), b), c). Это семейство является спектральной функиией самосопряженного оператора $A$ тогда и только тогда, когда: 1) для любого интервала $\Delta=[\alpha, \beta] E(\Delta) H$ приводит $A ; 2)$ если $f \in\left(E_{\beta+0}-E_{\alpha}\right) H,-\infty<\alpha<\beta<\infty$, то $\alpha\|f\|^{2} \leqslant(A f, f) \leqslant \beta\|f\|^{2}$.

2. Пусть $(X, \mu)$ - измеримое пространство с $\sigma$-конечной мерой $\mu$ такое, что гильбертово пространство $L^{2}(X, \mu)$ функций, модуль которых интегрируем в квадрате, сепарабельно. Мера $\mu$ определена на $\sigma$-алгебре подмножеств $X$, которая здесь не указывается. Далее везде пишется "измеримая", “почти всюду", “ $d \mu(x)$ " вместо соответственно “ $\mu$-измеримая", “ $\mu$-почти всюду", “ $\mu(d x)$ ”. Будем использовать следующие обозначения: $(\cdot, \cdot)$ и $\|\cdot\|$ - скалярное произведение и соответственно норма в $L^{2}(X, \mu)$, $l_{e}(x)=l_{e}-$ индикаторная функция множества $e \subset X, \Im(X, \mu)=\Im-$ множество всех измеримых на $(X, \mu)$ функций, $T_{\varphi}$ - оператор умножения на функцию $\varphi$ в $L^{2}(X, \mu)$, т.е. $T_{\varphi} f=\varphi f, f \in D(T)=\left\{f \in L^{2}(X, \mu): \varphi f \in L^{2}(X, \mu)\right\}$. Через $U$ обозначается замкнутьй (т. е. $\left.U^{2}=I\right)$ оператор в $L^{2}(X, \mu)$. Отметим следуюшие свойства таких операторов [3].

1) Оператор $U$ можно продолжить как операцию $U$, заданную на множестве $\Im(X, \mu)$ и сохраняющую свойство мультипликативности. Будем писать $U f=\check{f}$.

2) Существует измеримая почти всюду положительная функция $\varphi$, удовлетворяюшая условиям: $\check{\varphi}=\varphi^{-1}, U^{*}=T_{\varphi} U$, где сопряженньй к $U$ оператор $U^{*}$ определен на множестве $D\left(U^{*}\right)=\left\{f \in L^{2}(X, \mu): \varphi \check{f} \in L^{2}(X, \mu)\right\}$.

3) Максимальньй оператор $V=T_{\sqrt{\varphi}} U$ является унитарным и самосопряженным в $L^{2}(X, \mu)$. 
3. Пусть $\theta$ и $\eta$ - две измеримые на $(X, \mu)$ функции. Рассмотрим оператор

$$
T f=T_{\theta} f+T_{\eta} \check{f}, \quad U f=\check{f}, \quad f \in D(T)=\left\{f \in L^{2}(X, \mu): \theta f+\eta \check{f} \in L^{2}(X, \mu)\right\} .
$$

Согласно свойству 2) оператора $U$ существует измеримая, почти всюду положительная функция $\varphi(x)$ такая, что $U^{*}=T_{\varphi} U$, откуда $T^{*}=T_{\theta}+T_{\varphi} \overline{\bar{\eta}} U, D\left(T^{*}\right)=\left\{f \in L^{2}(X, \mu)\right.$ : $\left.\bar{\theta} f+\varphi \check{\bar{\eta}} \check{f} \in L^{2}(X, \mu)\right\}$. Оператор $T$ самосопряжен тогда и только тогда, когда

$$
\theta=\bar{\theta} \quad \text { и } \quad \eta=\varphi \check{\bar{\eta}} .
$$

Оператор $T$ ограничен тогда и только тогда, когда функции $|\theta|$ и $\sqrt{\varphi}|\check{\eta}|$ в существенном ограничены. В этом случае $\|T\| \leqslant\|\theta\|_{\infty}+\|\sqrt{\varphi} \check{\eta}\|_{\infty}$, где $\|f\|_{\infty}=\operatorname{vrai} \max |f(x)|$. Это утверждение легко следует из равенств, верных для любой функции $f \in L^{2}(X, \mu)$ :

$$
\begin{aligned}
\left\|T_{\eta} U f\right\|^{2} & =\left(T_{\eta} U f, T_{\eta} U f\right)=\left(f, U^{*} T_{|\eta|^{2}} U f\right)=\left(f, T_{\varphi|\check{\eta}|^{2}} f\right) \\
& =\left\|T_{\sqrt{\varphi}|\check{\eta}|} f\right\|^{2} \leqslant\|\sqrt{\varphi} \check{\eta}\|_{\infty}^{2}\|f\|^{2} .
\end{aligned}
$$

Применяя операцию $U$ к обеим частям уравнения $T g-\lambda g=f$, находим резольвенту $R_{\lambda}(T)$ оператора $T$ :

$$
g=R_{\lambda}(T) f=(\check{\theta}-\lambda) P^{-1}(\lambda) f-\eta P^{-1}(\lambda) \check{f}
$$

где $P(\lambda)=(\theta-\lambda)(\check{\theta}-\lambda)-\eta \check{\eta}$. Из $(1.2)$ следует, что $\lambda$ принадлежит резольвентному множеству оператора $T$ тогда и только тогда, когда функции $(\check{\theta}-\lambda) P^{-1}(\lambda)$ и $\sqrt{\varphi} \check{\eta} P^{-1}(\lambda)$ в существенном ограничены.

\section{2. Спектральная функция оператора $T$.}

1. Если $\varphi$ - вещественная измеримая функция на $(X, \mu)$, то оператор $T_{\varphi}$ умножения на $\varphi$ самосопряжен и его спектральная функция $E_{t}(\varphi), t \in \mathbb{R}$, есть семейство операторов умножения на $l_{e_{t}}, E_{t}(\varphi) f=l_{e_{t}} f, f \in L^{2}(X, \mu)$, где $e_{t}=\{x \in X: \varphi(x)<t\}$. Это легко следует из теоремы А.

Пусть $T$ - самосопряженньй оператор, поэтому условия (1.1) вьполнены.

Например, оператор $T=T_{\theta}+T_{\eta} U$ в $L^{2}(\mathbb{R})$, где $U f(x)=f\left(x^{-1}\right)\left(U^{*}=T_{x^{-2}} U\right)$ является самосопряженным, если $\theta$ вещественная, а $\eta(x)=x^{-1} g\left(x^{k}+x^{k}\right)$ с любыми $k \in \mathbb{N}$ и произвольной функцией $g(t)$, измеримой на $\mathbb{R}$.

Введем следующие обозначения: $\theta_{\mathrm{u}}=(\theta+\check{\theta}) / 2, \theta_{\mathrm{H}}=(\theta-\check{\theta}) / 2, G=\sqrt{\check{\varphi}|\eta|^{2}+\theta_{\mathrm{H}}^{2}}$, $\theta_{\mathrm{H}}^{0}=\theta_{\mathrm{H}} G^{-1}, \eta^{0}=\eta G^{-1}$. Мы предполагаем, что $G>0$ почти всюду. Для любого интервала $\Delta \subset \mathbb{R}$ положим

$$
e_{k}(\Delta)=\left\{x \in X: \theta_{\mathbf{u}}+(-1)^{k-1} G \in \Delta\right\}, \quad k=1,2 .
$$

В частности, при $\Delta=(-\infty, t)$ обозначим $e_{k}((-\infty, t))=e_{k}(t), k=1,2$.

ЛЕмма 1.1. Для любого интервала $\Delta \subset \mathbb{R}$

$$
\check{l}_{e_{k}(\Delta)}=l_{e_{k}(\Delta)}, \quad k=1,2 .
$$


ДоказАтельство. Оператор $T_{\varphi_{k}}$ умножения на $\varphi_{k}=\theta_{\mathrm{u}}+(-1)^{k-1} G, k=1,2$, самосопряжен, и его спектральная функция $E_{t}^{(k)}$ определяется равенством:

$$
E^{(k)}(\Delta) f=l_{e_{k}(\Delta)} f, \quad k=1,2 .
$$

Покажем, что $\check{G}=G$. Действительно, $\check{\theta}_{\mathrm{H}}^{2}=\theta_{\mathrm{H}}^{2}$ и, учитывая $(1.1)$ и равенство $\varphi \check{\varphi}=1$, имеем $\check{\varphi}|\eta|^{2}=\check{\varphi} \eta \bar{\eta}=\check{\varphi} \varphi \check{\bar{\eta}} \eta=\bar{\eta} \check{\eta}=\eta \check{\eta}$, откуда $\check{G}=G$ и $\check{\varphi}_{k}=\varphi_{k}, k=1,2$. Для любой $f \in L^{2}(X, \mu)$ выполнено равенство $U T_{\varphi_{k}} f=\varphi_{k} \check{f}=T_{\varphi_{k}} U f$. Таким образом, операторы $U$ и $T_{\varphi_{k}}$ перестановочны, следовательно, перестановочны операторы $U$ и $E^{(k)}(\Delta)$. Поэтому для любой $f \in L^{2}(X, \mu)$ вьполняется равенство: $\check{l}_{e_{k}(\Delta)} \check{f}=l_{e_{k}(\Delta)} \check{f}$, откуда $\check{l}_{e_{k}(\Delta)}=l_{e_{k}(\Delta)}, k=1,2$.

ТЕорема 1.1. Спектральная функиия $E_{t}, t \in \mathbb{R}$, самосопряженного оператора Т определяется равенством:

$$
E_{t} f=\Phi(t) f+\Psi(t) \check{f}, \quad f \in L^{2}(X, \mu),
$$

$2 \partial e$

$$
\Phi(t)=\frac{1}{2}\left[l_{e_{1}(t)}\left(1+\theta_{\mathcal{H}}^{0}\right)+l_{e_{2}(t)}\left(1-\theta_{\mathcal{H}}^{0}\right)\right], \quad \Psi(t)=\frac{\eta^{0}}{2}\left[l_{e_{1}(t)}-l_{e_{2}(t)}\right]
$$

ДокАЗАТЕЛЬСТво. Пусть $\Delta=[\alpha, \beta], E(\Delta)=E_{\beta+0}-E_{\alpha}, E(\Delta) f=\Phi(\Delta) f+\Psi(\Delta) \check{f}$, $f \in L^{2}(X, \mu)$, где

$$
\Phi(\Delta)=\frac{1}{2}\left[l_{e_{1}(\Delta)}\left(1+\theta_{\mathrm{H}}^{0}\right)+l_{e_{2}(\Delta)}\left(1-\theta_{\mathrm{H}}^{0}\right)\right], \quad \Psi(\Delta)=\frac{\eta^{0}}{2}\left[l_{e_{1}(\Delta)}-l_{e_{2}(\Delta)}\right] .
$$

Для доказательства теоремы согласно теореме А достаточно проверить следующие свойства оператора $E(\Delta): E(\Delta)$ - ортпроектор, $E_{-\infty}=O, E_{+\infty}=I$; если $\Delta \cap \Delta^{\prime}=\varnothing$, то подпространства $E(\Delta) L^{2}(X, \mu)$ и $E\left(\Delta^{\prime}\right) L^{2}(X, \mu)$ ортогональны; подпространство $E(\Delta) L^{2}(X, \mu)$ приводит $E(\Delta)$; если $\Delta=[\alpha, \beta]$, то для любой $f \in L^{2}(X, \mu)$ вьполнены оценки:

$$
\alpha\|E(\Delta)\|^{2} \leqslant(T E(\Delta) f, E(\Delta) f) \leqslant \beta\|E(\Delta) f\|^{2} .
$$

Эти свойства легко проверяются, учитывая лемму 1.1 и свойства оператора $U$.

1.3. Симметрические операторы умножения в $L^{2}(X, \mu)$.

1. Дадим схему построения симметрических операторов умножения в $L^{2}(X, \mu)$ с равными конечными дефектными числами.

Пусть $\psi$ - измеримая, вещественная, неограниченная функция, заданная на $(X, \mu)$ такая, что максимальньй оператор $T_{\psi}$ умножения на $\psi$ неограничен, самосопряжен и плотно определен, т. е. множество $D\left(T_{\psi}\right)=\left\{f \in L^{2}(X, \mu): \psi f \in L^{2}(X, \mu)\right\}$ плотно в $L^{2}(X, \mu)$.

Будем предполагать, что линейно независимые и измеримые на $(X, \mu)$ функции $a_{1}(x)$, $a_{2}(x), \ldots, a_{m}(x)$ удовлетворяют следующим условиям: $a_{j} \notin L^{2}(X, \mu)$ и $a_{j} /(\psi-\lambda) \in$ $L^{2}(X, \mu)$ для любых $j=1,2, \ldots, m$ и $\lambda \in \mathbb{C}, \operatorname{Im} \lambda \neq 0$. Определим линейное множество $H_{a}$ функций

$$
H_{a}=\left\{g \in \Im(X, \mu): g=f+\sum_{j=1}^{m} \alpha_{j} a_{j}, \quad f \in L^{2}(X, \mu), \quad \alpha_{j} \in C, \quad j=1,2, \ldots, m\right\}
$$


и оператор $Q$ проектирования из $H_{a}$ на $L^{2}(X, \mu)$. задаваемьй равенством $Q(f+$ $\left.\sum_{j=1}^{m} \alpha_{j} a_{j}\right)=f$.

Через $B_{\psi}$ будем обозначать линейный оператор в $L^{2}(X, \mu)$, определенньй на множестве $D\left(B_{\psi}\right)=\left\{f \in L^{2}(X, \mu): \psi f \in H_{a}\right\}$ равенством $B_{\psi} f=Q(\psi f)$. Так как $D\left(B_{\psi}\right) \supset D\left(T_{\psi}\right)$, то оператор $B_{\psi}$ плотно определен, следовательно, он имеет сопряженньй. Кроме того, легко установить, что оператор $B_{\psi}$ замкнут. Положим по определению $S_{\psi}=B_{\psi}^{*}$. Из включения $D\left(B_{\psi}\right) \supset D\left(T_{\psi}\right)$ следует равенство $D\left(B_{\psi}^{*}\right)=D\left(S_{\psi}\right) \subset$ $D\left(T_{\psi}^{*}\right)=D\left(T_{\psi}\right)$. Отсюда заключаем, что $S_{\psi}$ является замкнутым симметрическим оператором умножения на $\psi$ в пространстве $L^{2}(X, \mu)$. Покажем, что $S_{\psi}$ имеет индексы дефекта $(m, m)$. Для этого проверим, что функции

$$
\varphi_{j}(\lambda ; x)=\varphi_{j}(\lambda)=\frac{a_{j}}{\psi-\lambda}, \quad j=1,2, \ldots, m, \quad \operatorname{Im} \lambda \neq 0,
$$

принадлежат дефектному подпространству $\aleph_{\lambda}\left(S_{\psi}\right)=L^{2}(X, \mu) \ominus\left(S_{\psi}-\bar{\lambda} I\right) D\left(S_{\psi}\right)$ оператора $S_{\psi}$. Действительно,

$$
\begin{aligned}
\left(\left(S_{\psi}-\bar{\lambda} I\right) f, \varphi_{j}(\lambda)\right) & =\left(f, S_{\psi}^{*} \varphi_{j}(\lambda)-\lambda \varphi_{j}(\lambda)\right)=\left(f, Q\left[a_{j}+\lambda \varphi_{j}(\lambda)\right]-\lambda \varphi_{j}(\lambda)\right) \\
& =\left(f, \lambda \varphi_{j}(\lambda)-\lambda \varphi_{j}(\lambda)\right)=0
\end{aligned}
$$

Очевидно, что всякая функция из дефектного подпространства $\aleph_{\lambda}\left(S_{\psi}\right)$ оператора $S_{\psi}$ должна иметь вид (1.5) или являться линейной комбинацией таких функций.

2. Аналогично строятся симметрические операторы $S$ с индексами дефекта $(m, m)$ по самосопряженному оператору $T, T f=\theta f+\eta \check{f}$, содержащему инволюцию $U, U f=\check{f}$, и линейно независимой системе функций $a_{1}, a_{2}, \ldots, a_{m}$, удовлетворяющей условиям: $a_{j} \notin L^{2}(X, \mu)$

$$
\psi_{j}(\lambda)=(\check{\theta}-\lambda) P^{-1}(\lambda) a_{j}-\eta P^{-1}(\lambda) \check{a}_{j} \in L^{2}(X, \mu), \quad j=1,2, \ldots, m, \quad \operatorname{Im} \lambda \neq 0 .
$$

Сохраняя прежние обозначения, определим линейный оператор на линеале $D(B)=\{f \in$ $\left.L^{2}(X, \mu): \theta f+\eta \check{f} \in H_{a}\right\}$ равенством $B f=Q(\theta f+\eta \check{f})$. Положим $S=B^{*}$. Оператор $S$ является симметричным с индексами дефекта $(m, m)$, функции $\psi_{j}(\lambda), j=1,2, \ldots, m$, определенные условием (1.6), принадлежат дефектному подпространству $\aleph_{\lambda}(S)$ оператора $S$.

\section{2. Спектральные функции операторов типа свертки в пространстве $L^{2}\left(\mathbb{R}^{n}\right)$}

\section{1. Операторы типа свертки.}

1. Через $S\left(\mathbb{R}^{n}\right)$ обозначим пространство Шварца бесконечно дифференцируемых комплекснозначных функций $\varphi(x), x \in \mathbb{R}^{n}$, таких, что

$$
\sup _{x \in \mathbb{R}^{n}}\left|x^{\beta}\left(D^{\alpha}\right) \varphi(x)\right|<\infty
$$

для всех $\alpha, \beta$ из $\mathbb{N}^{n}$ с топологией, определяемой этой полунормой. Здесь $\mathbb{N}^{n}$ - множество мультииндексов $\alpha=\left(\alpha_{1}, \ldots, \alpha_{n}\right), \alpha_{i} \in \mathbb{N}, x^{\beta}=x_{1}^{\beta_{1}} \ldots x_{n}^{\beta_{n}}, D^{\alpha}=\partial^{\alpha} / \partial x_{1}^{\alpha_{1}} \ldots \partial x_{n}^{\alpha_{n}}$, $|\alpha|=\alpha_{1}+\cdots+\alpha_{n}$. Двойственное к $S\left(\mathbb{R}^{n}\right)$ пространство обозначается $S^{\prime}\left(\mathbb{R}^{n}\right)$, а его 
элементы назьваются медленно растущими обобщенными функииями. Через $F$ обозначим преобразование $Ф$ урье $S\left(\mathbb{R}^{n}\right)$ :

$$
(F \varphi)(x)=\widehat{\varphi}(x)=\int_{\mathbb{R}^{n}} e^{-2 \pi i x t} \varphi(t) d t, \quad \varphi \in S\left(\mathbb{R}^{n}\right),
$$

распространенное на все $f \in S^{\prime}\left(\mathbb{R}^{n}\right)$ по непрерывности или, что эквивалентно, по формуле $\langle\widehat{f}, \varphi\rangle=\langle f, \bar{F} \varphi\rangle$, где $\langle f, \varphi\rangle$ - значение функционала $f$ на $\varphi, \bar{F} \varphi=\widehat{\varphi}(-x)$ - обратное преобразование $Ф$ урье.

Известно [4], что преобразование Фурье изоморфно отображает пространства $S\left(\mathbb{R}^{n}\right)$ и $S^{\prime}\left(\mathbb{R}^{n}\right)$ на себя, а в $L^{2}\left(\mathbb{R}^{n}\right)$ оно является унитарным оператором.

Отметим, что любая измеримая и ограниченная на $\mathbb{R}^{n}$ функция есть элемент $S^{\prime}\left(\mathbb{R}^{n}\right)$. Через $S_{1}^{\prime}\left(\mathbb{R}^{n}\right)$ обозначим множество обобщенных функций $f \in S^{\prime}\left(\mathbb{R}^{n}\right)$, преобразование Фурье которых $\widehat{f}$ есть обычная функция, локально интегрируемая в квадрате, т. е. $\widehat{f} \in$ $L_{\text {loc }}^{2}\left(\mathbb{R}^{n}\right)$.

2. Пусть $\xi, \zeta \in S_{1}^{\prime}\left(\mathbb{R}^{n}\right), V$ - замкнутьй инволютивный оператор в $L^{2}\left(\mathbb{R}^{n}\right)$ такой, что $U=F V \bar{F}$ есть мультипликативная инволюция. Например, если $V f(x)=e^{2 \pi i a x} f(-x)$, то $U f(x)=f(a-x)$.

Назовем оператором типа свертки в $L^{2}\left(\mathbb{R}^{n}\right)$ всякий оператор $\widetilde{A}$, определенньй на линеале $D(\widetilde{A}) \subset\left\{f \in L^{2}\left(\mathbb{R}^{n}\right): \xi * f+\eta *(V f) \in L^{2}\left(\mathbb{R}^{n}\right)\right\}$. Положим $\widehat{\xi}=\theta, \widehat{\zeta}=\eta$. Оператор $T=F A \bar{F}, T f=\theta f+\eta \check{f}$, определен на $D(T)=\left\{f \in L^{2}\left(\mathbb{R}^{n}\right): \theta f+\eta \check{f} \in L^{2}\left(\mathbb{R}^{n}\right)\right\}$. Здесь $\check{f}=U f, U=F V \bar{F}$. Оператор $\widetilde{T}=F V \widetilde{F}$ определен на множестве $D(\widetilde{T}) \subset D(T)$.

Будем предполагать, что операторы $T$ и $A$ - самосопряженные, и обозначим через $E_{t}(T), E_{t}(A), t \in \mathbb{R}$, их спектральные функции.

Теорема 2.1. Спектральная функиия $E_{t}(A), t \in \mathbb{R}$, оператора $A$ определяется выражением

$$
E_{t}(A) f=\bar{F} \Phi * f+\bar{F} \Psi *(V f), \quad f \in L^{2}\left(\mathbb{R}^{n}\right),
$$

где функиии $\Phi$ и определены равенствами (1.4).

ДокАЗАТЕЛЬСТво. Из унитарной эквивалентности операторов $A$ и $T$ следует, что их спектральные функции связаны соотношением $E_{t}(A)=\bar{F} E_{t}(T) F$. Учитывая, что спектральная функция $E_{t}(T), t \in \mathbb{R}$, задана равенством (1.3), приходим к (2.1).

\section{2. Частные случаи.}

1. Если $\zeta=0, \xi \in S_{1}^{\prime}\left(\mathbb{R}^{n}\right)$, то $A f=\xi * f$. Резольвента оператора $A$ определяется равенством

$$
(A f-\lambda I)^{-1} f=R_{\lambda}(A) f=\bar{F}\left[(\widehat{\xi}-\lambda)^{-1}\right] * f
$$

для всех $\lambda$, не принадлежащих существенной области значений $\widehat{\xi}$. Оператор $A$ самосопряжен тогда и только тогда, когда $\widehat{\xi}$ вещественна, его спектральная функция в этом случае задается [5] равенством $E_{t}(A) f=\widehat{l}_{e_{t}} * f, t \in \mathbb{R}, e_{t}=\left\{x \in \mathbb{R}^{n}: \widehat{\xi}(x)<t\right\}$, $f \in L^{2}\left(\mathbb{R}^{n}\right)$.

Рассмотрим обобщенную функцию $\xi=P_{k}(\delta)=\sum_{|\alpha| \leqslant k} a_{\alpha} D^{\alpha} \delta$, где $k \in \mathbb{N}, \alpha \in \mathbb{N}^{n}$, $a_{\alpha}$-постоянные, $\delta$ - дельта-функция Дирака. Оператор $A f=\xi * f=\sum_{|\alpha| \leqslant k} a_{\alpha} D^{\alpha} f$, $f \in D(A)$, есть дифференциальньй оператор с постоянньми коэффициентами. Например, операторы $\Delta f=\sum_{j=1}^{n} \partial^{2} f / \partial t_{j}^{2}, \square f=\partial^{2} f / \partial t^{2}-\Delta f$ являются самосопряженными операторами свертки. 
Максимальный оператор свертки с $\xi=g * P_{k}(\delta)$, где $g \in S_{1}^{\prime}\left(\mathbb{R}^{n}\right)$, является интегро-дифференциальным. Он будет самосопряженным тогда и только тогда, когда функция $\widehat{\xi}=\widehat{g} \cdot P_{k}(2 \pi i x)$ вещественна.

2. Если $\xi, \zeta \in L^{2}\left(\mathbb{R}^{n}\right)$, а оператор $V$ определен равенством $V f(x)=f\left(\Lambda_{\alpha} x\right), f \in$ $L^{2}\left(\mathbb{R}^{n}\right)$, где $\Lambda_{\alpha}, \alpha=\left(\alpha_{1}, \ldots, \alpha_{n}\right) \in \mathbb{N}^{n},-$ диагональная матрица с главной диагональю $(-1)^{\alpha_{1}}, \ldots,(-1)^{\alpha_{n}}$, то $A$ есть интегральньй оператор Карлемана с ядром, зависящим от разности и суммы аргументов, т. е.

$$
A f(x)=\int_{\mathbb{R}^{n}}\left[\xi(x-y)+\zeta\left(x-\Lambda_{\alpha} y\right)\right] f(y) d y .
$$

Спектральные функции всех указанных операторов определяются теоремой 2.1.

\section{3. Симметрические операторы свертки в $L^{2}\left(\mathbb{R}^{n}\right)$.}

1. Ради простоты изложения в этом пункте будут рассматриваться симметрические операторы с индексами дефекта $(m, m)$ в пространстве $L^{2}(\mathbb{R})$, хотя аналогичные операторы можно рассматривать в пространствах $L^{2}\left(\mathbb{R}^{n}\right), n>1$.

Через $\AA$ обозначим максимальный самосопряженньй оператор свертки, $\AA f=\xi * f$, где $\xi \in S_{1}^{\prime}(\mathbb{R})$ такая, что $\widehat{\xi}$ - вещественная функция. Оператор $\AA$ унитарно эквивалентен максимальному оператору $T_{\psi}$ умножения на $\psi=\widehat{\xi}, T_{\psi}=\bar{F} \AA$ F.

Если $S_{\psi}=S$ - симметричньй оператор умножения с индексами дефекта $(m, m)$, построенный по функциям $a_{1}(x), a_{2}(x), \ldots, a_{m}(x)$ в пункте 1.3 , то оператор $A=F S \bar{F}$ будет также симметричным с теми же индексами дефекта. При этом функции $\varphi_{j}(\lambda)=$ $F\left[a_{j}(\psi-\lambda)^{-1}\right], j=1,2, \ldots, m$, составляют базис дефектного подпространства $\aleph_{\lambda}$ оператора $A$. Отметим, что область определения $D(A)$ оператора $A$ есть линеал

$$
D(A)=\left\{f \in L^{2}(\mathbb{R}): \xi * f \in L^{2}(\mathbb{R}), \quad \int_{-\infty}^{\infty} a_{j}(x) \widehat{f}(x) d x=0, \quad j=1,2, \ldots, m\right\} .
$$

Рассмотрим случай, когда $\xi=P_{k}(\delta), k \geqslant m$. В этом случае $\AA$ - максимальный дифференциальньй оператор в пространстве $L^{2}(\mathbb{R})$ с постоянными коэффициентами, $\widehat{\xi}=$ $P_{k}(2 \pi i x)$ - многочлен степени $k$. Беря в качестве системы функций $a_{1}, a_{2}, \ldots a_{m}$ систему $1, x, x^{2}, \ldots, x^{m-1}$, получаем для любого $j=0,1, \ldots, m-1 x^{j}[\psi(x)-\lambda]^{-1} \in L^{2}(\mathbb{R})$, $\operatorname{Im} \lambda \neq 0$.

Область определения $D(A)$ оператора $A$ определяется как множество функций $f \in$ $L^{2}(\mathbb{R}), m-1$ раз дифференцируемых таких, что $\xi * f \in L^{2}(\mathbb{R})$, и удовлетворяющих условиям: $f(0)=f^{\prime}(0)=\cdots=f^{(m-1)}(0)=0$.

Подробно рассмотрим операторы дифференцирования первого и второго порядков в $L^{2}(\mathbb{R})$.

2. Рассмотрим $\xi=(2 \pi i)^{-1} \delta$. Тогда $\widehat{\xi}=x$. Максимальньй оператор $\AA$ есть оператор дифференцирования первого порядка, $\AA=f^{\prime} /(2 \pi i)$, он унитарно эквивалентен максимальному оператору $T_{x}$ умножения на $x$. Симметричньй оператор $A$, определяемый равенством $A f=f^{\prime} /(2 \pi i)$ на всех функциях $f \in D(\AA)$, удовлетворяющих условию $f(0)=0$, имеет индексы дефекта $(1,1)$. Отметим, что если $f \in D(\AA)$, то в точке 0 она может иметь конечный скачок.

Применяя теорию вычетов к вычислению интегралов, находим элементы дефектных подпространств $\varphi_{\lambda}(x)=(2 \pi i)^{-1} F\left[(x-\lambda)^{-1}\right]$. Имеем

$$
\varphi_{\lambda}(x)=l_{\varepsilon(\lambda)}(x) e^{2 \pi i \lambda x}, \quad \operatorname{Im} \lambda \neq 0
$$


где $l_{\varepsilon(\lambda)}(x)=l_{+}(x)=l_{(0, \infty)}(x)$, если $\operatorname{Im} \lambda>0$, и $l_{\varepsilon(\lambda)}(x)=l_{-}(x)=l_{(-\infty, 0)}(x)$, если $\operatorname{Im} \lambda<0$, есть индикаторные функции соответственно множеств $(0, \infty)$ и $(-\infty, 0)$.

Пусть $\stackrel{\circ}{R}_{\lambda}=(\AA-\lambda I)^{-1}$ - резольвента самосопряженного оператора $\AA$. Множество всех обобщенных резольвент оператора $A$ (т. е. резольвент всех квазисамосопряженных расширений оператора $A$ ) описывается формулой [4]:

$$
R_{\lambda} f=\stackrel{\circ}{R}_{\lambda} f+\frac{1-\omega(\lambda)}{\omega(\lambda) C(\lambda)-1} \cdot \frac{\left(f, \varphi_{\bar{\lambda}}\right)}{(\lambda+i)\left(\varphi_{\lambda}, \varphi_{i}\right)} \varphi_{\lambda}, \quad \operatorname{Im} \lambda>0,
$$

где $\omega(\lambda)$ - произвольная аналитическая в верхней полуплоскости функция, не превосходящая по модулю единицы, $|\omega(\lambda)| \leqslant 1, C(\lambda)=((\lambda-i) /(\lambda+i)) \cdot\left(\left(\varphi_{\lambda}, \varphi_{-i}\right) /\left(\varphi_{\lambda}, \varphi_{-i}\right)\right)$, $\operatorname{Im} \lambda>0,-$ характеристическая функция оператора $A$. Оператор $R_{\lambda}$ является резольвентой некоторого самосопряженного расширения оператора $A$ тогда и только тогда, когда $\omega(\lambda)=z=$ Const, $|z|=1$.

Учитывая (2.2) и (2.3), при условии $\operatorname{Im} \lambda>0$ запишем (2.4) в виде

$$
\left(R_{\lambda} f\right)(x)=\left(r_{l} * f\right)(x)+\frac{i}{2 \pi}[1-\omega(\lambda)]\left(\int_{-\infty}^{0} f(x) e^{-2 \pi i \bar{\lambda} x} d x\right) l_{+}(x) e^{2 \pi i \lambda x}
$$

где $r_{l}(x)=\bar{F}\left[(x-\lambda)^{-1}\right]=2 \pi i l_{+}(x) e^{2 \pi i x \lambda}$. Таким образом,

$$
\begin{aligned}
\left(R_{\lambda} f\right)(x)= & 2 \pi i e^{2 \pi i x \lambda} \int_{x}^{\infty} e^{-2 \pi i t \lambda} f(t) d t \\
& -2 \pi i[1-\omega(\lambda)] \cdot\left(\int_{-\infty}^{0} f(x) e^{-2 \pi i \bar{\lambda} x} d x\right) \cdot l_{+}(x) e^{2 \pi i \lambda x} .
\end{aligned}
$$

Обобщенная спектральная функция $E_{t}$ оператора $A$ связана с его обобщенной резольвентой $R_{\lambda}$ формулой

$$
R_{\lambda} f=\int_{-\infty}^{\infty} \frac{d E_{t} f}{t-\lambda}, \quad f \in L^{2}(\mathbb{R}) .
$$

3. Рассмотрим $\xi=-\delta^{\prime \prime} /\left(4 \pi^{2}\right), \widehat{\xi}=x^{2}$. В этом случае оператор $\AA$ есть дифференциальньй оператор второго порядка в $L^{2}(\mathbb{R}), \AA f=-f^{\prime \prime} /\left(4 \pi^{2}\right)$. Оператор $B$, определенный на $D(B)=\left\{f \in D(\AA): f^{\prime}=0\right\}$ выражением $B f=-f^{\prime \prime} /\left(4 \pi^{2}\right), f \in D(B)$, является симметричным с индексами дефекта $(1,1)$. Функция $\varphi_{\lambda}(x)=e^{2 \pi i \sqrt{\lambda}|x|}, \operatorname{Im} \sqrt{\lambda}>0$, принадлежит дефектному подпространству $\aleph_{\bar{\lambda}}$.

\section{СПИСОК ЦИТИРОВАННОЙ ЛИТЕРАТУРЫ}

[1] Соболев В.В. Курс теоретической астрофизики. М.: Наука, 1967.

[2] Ахиезер Н.И., Глазман И. М. Теория линейных операторов в гильбертовом пространстве. М.: Наука, 1966.

[3] Александров Е. Л. Операторы в пространствах $L^{2}(X, \mu)$ и некоторые их приложения. Деп. в ВИНИТИ 14.04.97 № 1245-В97,. Саратов, 1997.

[4] Vo-Khac-Khoan. Distribution, Analyse de Fourier. Operateurs aux derivées partielles. Vuibert, 63, Bd. Saint Germain. V. 1, 2. Paris, 1967.

[5] Александров Е. Л. Спектральные свойства операторов с ядрами, зависящими от разности аргументов // Изв. вузов. Матем. 1991. № 8 (351). С. 3-8.

[6] Александров Е. Л., Ильмушкин Г. М. Обобщенные резольвенты изометрических и симметрических операторов // Изв. вузов. Матем. 1977. №1(176). С. 15-25. 